\title{
La educación universitaria en el bolsillo, aplicaciones y entornos virtuales
}

\author{
Héctor MOLINA GARCÍA \\ Universidad Francisco de Vitoria \\ h.molina@ufv.es
}

Recibido: $11 / 10 / 2012$

Aceptado: 23/01/2013

\begin{abstract}
Resumen
Es una realidad que en la actualidad existe una tendencia hacia nuevas formas de educar en las universidades españolas, el nuevo Espacio Europeo de Educación Superior y la aparición y desarrollo de las nuevas tecnologías móviles de entornos compartidos o del denominado video en streaming, han hecho posible este diferente escenario. Tanto el educador como el educando se han percatado de esta tendencia y tratan de adaptar y aprovechar los recursos en el génesis de este contexto. Esta investigación trata de descifrar los nuevos paradigmas relativos a las nuevas herramientas de carácter móvil que se utilizan en las aulas de las universidades españolas.
\end{abstract}

Palabras clave: Tecnología móvil, Educación, Fórmulas Docentes, Nuevas Tecnologías.

\section{The university's education in the pocket, applications and virtual environments}

\begin{abstract}
It is a fact that nowadays, there is a trend towards new forms of education in the Spanish universities, the new European Higher Education Area and the emergence and development of new mobile technologies with shared environments or the so called streaming video are the ones who made possible this different scenario. Both, the educator and the learner, have noticed this trend and try to adapt and use the resources in the genesis of this context. This research tries to decipher the new paradigms on emerging mobile character tools used in the classrooms of the Spanish universities.
\end{abstract}

Keywords: Mobile Technology, Education, Teachers Formulas, New Technologies.

\section{Referencia normalizada}

MOLINA GARCÍA, Héctor (2013): "La educación universitaria en el bolsillo, aplicaciones y entornos virtuales". Estudios sobre el Mensaje Periodístico. Vol. 19, Núm. especial marzo, págs.: 319-328. Madrid, Servicio de Publicaciones de la Universidad Complutense.

Sumario: 1. Introducción. 2. Metodología. 3. Desarrollo. 3.1. El Smartphone como propuesta docente. 3.2. Los ambientes educativos virtuales, una apuesta universitaria. 3.3. Aplicaciones de carácter educativo en las universidades españolas. 4. Conclusiones. 5. Referencias bibliográficas.

\section{Introducción}

En la actualidad, las nuevas Tecnologías de la Información están más presentes que nunca en las Universidades Europeas. Este hecho, quizá esté determinado por dos acontecimientos bien diferenciados. Por un lado, la aparición y desarrollo de dispositivos móviles con la suficiente potencia como para poder instalar aplicaciones y que estén conectados a Internet, y por otro lado, las directivas marcadas desde el nuevo Espacio Europeo de Educación Superior.

Una de las particularidades de esta nueva realidad, es que el desarrollo, en parte, ha surgido desde abajo hacia arriba, los cambios en la tecnología han sido absorbidos en primer lugar por los alumnos, y con posterioridad, los docentes y las instituciones 
se han dado cuenta del potencial de estas nuevas herramientas, y ahora, tratan de adaptarlas en sus disciplinas. Este es el caso de los dispositivos móviles, que han pasado de ser un obstáculo en la comunicación y en el desarrollo de las clases, a formar parte de las dinámicas y competencias propias de las asignaturas.

Los nuevos entornos educativos multimedia no sólo deben reproducir la realidad de las aulas, sino que además tienen que mejorarlas y aportar nuevos conceptos. Por ejemplo, se pueden crear grupos de discusión, resolución de prácticas en común, creación de Wikis con terminología propia de la asignatura, etc.

\section{Metodología}

Esta labor investigadora intenta interpretar y comprender los nuevos modelos relativos a las nuevas herramientas de carácter móvil que se utilizan en las aulas de las universidades españolas, para ello, esta investigación se apoya en dos pilares fundamentales, un marco teórico, en el que se analizan todos los conocimientos existentes sobre el objeto de estudio; y un trabajo de campo, en el que se recoge información de los principales instrumentos de carácter móvil que actualmente se utilizan en las universidades españolas. Se han estudiado todas las páginas web de las universidades españolas para tratar de describir, catalogar y analizar los entornos virtuales de los que disponen, y los desarrolladores de los mismos, tanto de universidades públicas como privadas (Ministerio de Educación, Cultura y Deporte, 2012). Además, se han buscado en los dos mercados principales de aplicaciones para móviles (Markets) el software institucional de las Universidades españolas disponible, y el resultado ha sido muy esclarecedor. Lógicamente, sólo se han tenido en cuenta aplicaciones institucionales desde las que los alumnos puedan realizar labores docentes.

Este estudio ofrece un retrato robot del panorama de la educación en universidades mediante dispositivos móviles y brinda una nueva propuesta en la que convergen las nuevas tecnologías móviles con los medios tradicionales para lograr los objetivos competenciales.

\section{Desarrollo}

Las nuevas tecnologías en el ámbito de la educación, han sufrido transformaciones que a grandes rasgos han supuesto un incremento de la portabilidad, una conexión permanente, una reducción del tamaño de los instrumentos, y un aumento en la potencia y velocidad de procesado. En este sentido, actualmente, el máximo exponente es el Smartphone, que es una evolución del teléfono móvil.

\subsection{EI Smartphone como propuesta docente}

La implantación y estandarización de los teléfonos móviles supuso en un principio un claro perjuicio para la labor docente, ya que la interrupción constante de las clases, a causa de llamadas y mensajes, era y es, muy molesto e impide una transmisión de conocimientos con las mínimas garantías, pero la implantación y desarrollo es inevitable.

En el mercado existe gran cantidad de teléfonos Smartphone, pero la realidad es que está liderado por las compañías Apple y Samsung, según el analista de UBS, May- 
nard Um estas dos compañías representan más del 55\% de los ingresos de la industria y más del 90\% del EBIT total (Zach Epstein, 2012).

Según el informe anual sobre el desarrollo de la sociedad de la información en España 2011 eEspaña (Fundación Orange, 2011: 12) en los últimos tres años 46 países se sumaron a ofrecer a sus ciudadanos servicios de tecnología $3 \mathrm{G}$, lo que hace que un total de 143 países dispongan de esta tecnología. En el año 2010 se han superado los 2.000 millones de usuarios de internet, de los que un $60 \%$ se encuentran en países en vías de desarrollo.

En este mismo informe (Fundación Orange, 2011: 15) se detalla como en los últimos años ha habido un incremento sustancial de la venta de teléfonos móviles, casi en paralelo a la penetración de la banda ancha en telefonía móvil. En cambio, el índice de penetración de telefonía y banda ancha fija han descendido. Estos datos no están aislados, ya que hay numerosos informes que ratifican estos mismos hechos. Según el informe de la Sociedad de la Información en España 2011 siE[11 de la Fundación Telefónica (Fundación Telefónica, 2011: 14) la banda ancha móvil ha experimentado un crecimiento semestral superior al 18\%. Estos datos estadísticos nos muestran una fotografía de la realidad muy evidente, el perfil del usuario de internet está cambiando, los usuarios actuales, no sólo están conectados en sus casas, en sus centros de trabajo o en las universidades, sino que también disponen de conexión en sus bolsillos, en sus dispositivos móviles.

Según el informe trimestral Gartner (Gartner, 2012) en el cuarto trimestre de 2011 las ventas de Smartphones han sufrido un incremento de un 47,3\% respecto al mismo periodo del año anterior. La compañía Apple ha duplicado las ventas y Samsung incrementa su cuota de forma más moderada.

En el marco educacional se pueden enumerar las principales particularidades y rasgos potenciales que tienen este tipo de dispositivos:

» Acceso a Internet. Los Smartphone son vendidos por las operadoras con tarifas de conexión a internet de banda ancha. Las ventajas que ofrece estar conectado a la red en todo momento son numerosas y posibilita por ejemplo:

- Conexión y sincronización inmediata con el correo electrónico, calendario, notas y contactos. Tanto alumnos, como profesores llevan en el bolsillo una herramienta ofimática capaz de ponerles en contacto entre sí y de gestionar su agenda y contactos.

- Navegación por la web, realizar consultas, resolución de dudas, consultar fuentes bibliográficas, etc. En este sentido, los ejemplos educativos son innumerables.

- Visualización de vídeos y televisión en streaming. Con este tipo de dispositivos, el docente puede enviar el material audiovisual a los alumnos y estos verlo en cualquier momento a través de sus teléfonos.

- Hablar y escribir a los contactos de forma gratuita. Esta es quizá una de las peculiaridades que ha fomentado el crecimiento exponencial de los Smartphone entre los jóvenes.

- Conexión y sincronización con todas las redes sociales. 
» Es una cámara fotográfica y de vídeo de alta definición. Por ejemplo, el recientemente aparecido iPhone 5 dispone de una cámara con una calidad de 8 Mpx.

» Puede ser una potente consola de juegos portátil. Según un estudio de la compañía Flurry los videojuegos para móviles suponen el 8\% de mercado estadounidense.

»Permite descargar aplicaciones de todo tipo. Existe cerca de un millón de aplicaciones distintas que se pueden descargar en un Smartphone (Buengeek 2011).

» Dispone de localización mediante satélites GPS y su aplicación en las redes sociales y navegadores GPS.

» Son buenos reproductores de música portátiles, con gran calidad y capacidad.

» Lectura y edición de todo tipo de documentos. (función más utilizada en tabletas, ya que la visualización en Smartphone puede resultar poco legible e incómoda). Los estudiantes tienen acceso en cualquier lugar y en cualquier momento a la información que el profesor deje alojado en el espacio virtual de la asignatura.

En este sentido son numerosas las aplicaciones que se pueden promover dentro del aula y que cubrirían las necesidades de alguna de las competencias asignadas para cada asignatura.

\subsection{Los ambientes educativos virtuales, una apuesta universitaria}

Como se ha analizado con anterioridad, las Universidades apuestan cada vez más por adaptar las nuevas tecnologías a la enseñanza. Existe una tendencia creciente que ya vaticinó Daniel (Daniel, 1996) que consiste en la creación de Mega-Universidades en toda Europa, que ofrecen gran cantidad de recursos tanto a sus alumnos como a sus profesores. El caso español es un claro ejemplo de esta evolución. A mediados de los años setenta existía poco más de medio millón de jóvenes que cursaran estudios universitarios, actualmente esta cifra es de casi un millón y medio de estudiantes matriculados (INE, 2011), sin contar con los cursos de postgrado.

Por esta razón, el gran desafío en el que se encuentran las universidades españolas es el de atender correctamente a esta demanda tan amplia, incrementando la calidad docente y apostando con mayor ímpetu en la producción científica de sus profesores. Para ello, se ponen en marcha los denominado espacios virtuales educativos. El profesor Manuel Area Moreira (Area, 2001) cree que este espacio educativo virtual puede servir para el desarrollo de dos grandes funciones pedagógicas bien diferenciadas:

Por un lado, la red como apoyo a la docencia presencial. Este tipo de entornos ofrecen materiales y recursos docentes que pueden servir de sostén para las clases universitarias presenciales. Gracias a estas herramientas se pueden complementar las clases tradicionales con la utilización de las nuevas tecnologías. En este sentido, el denominado campus virtual, es un complemento de la actividad y organización docente en las universidades.

Por otro lado, la red como escenario para la educación a distancia. Estas herramientas se pueden utilizar para ofrecer una modalidad de enseñanza a distancia, con 
lo que se accede a sectores de la población que normalmente no pueden formarse directamente en los campus universitarios, por ejemplo, por motivos laborales.

La realidad es que se podrían definir los espacios virtuales educativos desde el punto de vista de los usuarios: alumnos y profesores.

Para los alumnos, un campus virtual es un espacio en el que debe estar todo organizado, y se debe acceder a los contenidos de forma flexible. Además, es un espacio institucional en el que los recursos están disponibles las 24 horas del día los 365 del año.

Para el personal docente, además de adquirir las ventajas de los alumnos (espacio organizado, flexible, institucional y permanente), es un lugar en el que interactuar con los alumnos de forma personalizada o colectiva.

En la actualidad todas las universidades españolas mantienen campus virtuales que ponen a disposición de la comunidad académica. Para esta labor investigadora se han visitado todas las páginas web de todas las universidades españolas para tratar de describir, catalogar y analizar los entornos virtuales de los que disponen y los desarrolladores de los mismos, tanto de universidades públicas como privadas (Ministerio de Educación, Cultura y Deporte 2012).

Los resultados obtenidos han sido rotundos, actualmente las 77 universidades españolas, públicas y privadas, disponen de entornos virtuales desde los que ofrecen multitud de servicios destinados a la labor docente, desde compartir archivos, hasta la integración de herramientas avanzadas de e-learning.

Respecto a las plataformas sobre las que están creadas estas aulas virtuales existen fundamentalmente dos de ellas que suman $77 \%$ de universidades, Moodle y Blackboard. El resto cuentan con desarrollos propios u otras plataformas comerciales como Sakai, CampusNet o Atnova. Algunas universidades ofrecen incluso tres plataformas para entornos educativos virtuales, es el caso de la Universidad Complutense de Madrid, en la que se han desarrollado campus virtuales en Moodle, Blackboard y Sakai.

1. Plataformas de los entornos virtuales de las Universidades españolas (Elaboración propia)

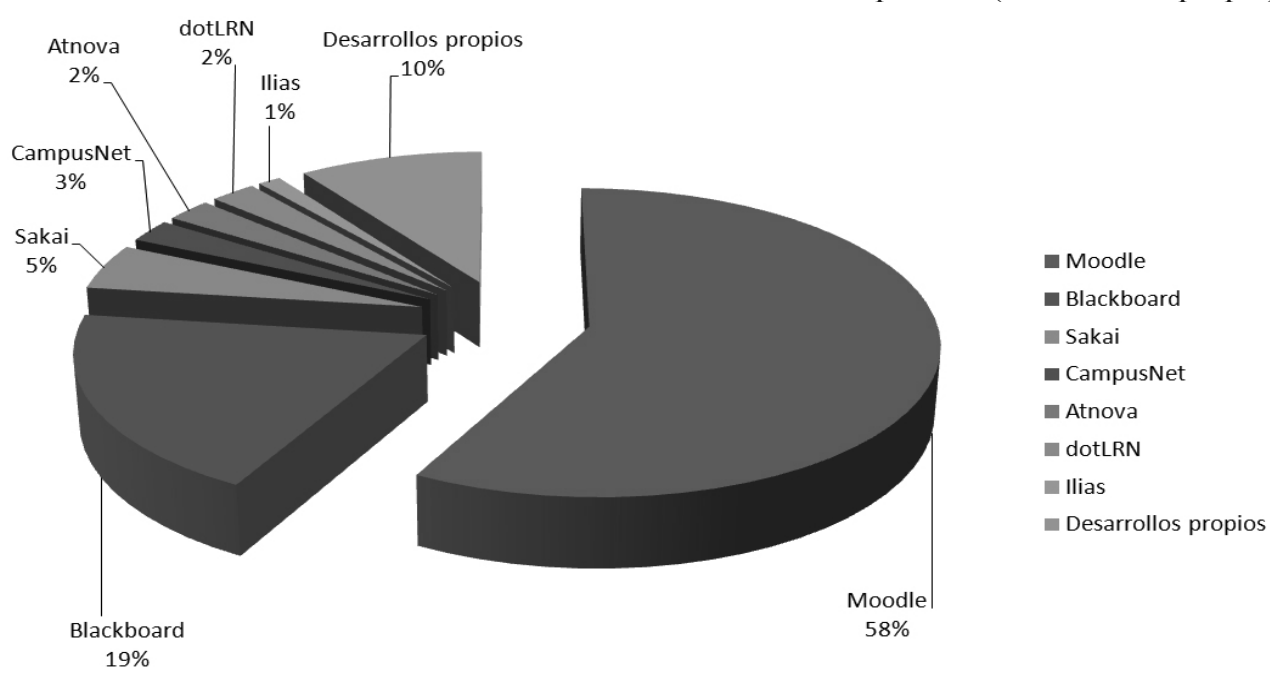


Según el gráfico mostrado, el 58\% de las universidades españolas disponen de un entorno educativo creado en Moodle, mientras que un 19\% utilizan como plataforma Blackboard. La principal diferencia entre ambas es que Moodle está creado mediante código abierto y su uso y distribución es completamente libre, mientras que Blackboard es un sistema propietario y los usuarios tienen que comprar licencias de uso.

Otra diferencia importante es que Moodle dispone de un soporte técnico comunitario completamente gratuito, mientras que Blackboard requiere el abono de una cuota anual para este mismo servicio.

La realidad es que ambas plataformas disponen de herramientas similares para los usuarios que son muy útiles para que los alumnos adquieran las competencias de cada materia: chats, bases de datos, cuestionarios, encuestas, foros, glosarios, etc.

Es un hecho que las universidades españolas trabajan desde hace años con este tipo de entornos y cada día se innova introduciendo nuevas herramientas que se aplican inmediatamente en la vida académica.

\subsection{Aplicaciones de carácter educativo en las universidades españolas}

La evolución natural de estos entornos educativos estudiados, se encamina inevitablemente a que se instalen en los Smartphone de los alumnos y de los profesores, y que todas las actividades académicas se gestionen desde estos pequeños dispositivos.

Para esta labor investigadora se han buscado en los dos mercados principales de aplicaciones para móviles (Markets) el software institucional de las Universidades españolas disponible, y el resultado ha sido muy esclarecedor. Lógicamente, sólo se han tenido en cuenta aplicaciones institucionales desde las que los alumnos puedan, desde consultar sus notas, hasta disponer de un espacio para sus asignaturas.

Por un lado, se ha buscado en el App Store (2012) de la compañía Apple para los dispositivos con el sistema operativo IOS. Actualmente 20 universidades españolas cuentan con aplicaciones que son adaptaciones de sus campus virtuales para los dispositivos de Apple:

2. Universidades españolas con aplicaciones de entornos educativos para el sistema operativo IOS (Elaboración propia)

\begin{tabular}{|l|l|}
\hline Alicante & Navarra \\
\hline Autónoma de Barcelona & Oviedo \\
\hline Autónoma de Madrid & Politécnica de Catalunya \\
\hline Barcelona & Pontificia Comillas \\
\hline Carlos III de Madrid & Pública de Navarra \\
\hline Córdoba & Sevilla \\
\hline Europea de Madrid & U. a Distancia de Madrid \\
\hline Granada & Valencia \\
\hline Int. Menéndez Pelayo & Valladolid \\
\hline Murcia & Zaragoza \\
\hline
\end{tabular}


Por otro lado, se ha buscado en el Google Play (2012), la tienda de aplicaciones de Android, las aplicaciones de entornos educativos de las universidades españolas, y el resultado ha sido muy parecido al anterior.

3. Universidades españolas con aplicaciones de entornos educativos para el sistema operativo Android (Elaboración propia)

\begin{tabular}{|l|l|}
\hline Alfonso X el Sabio & Int. Menéndez Pelayo \\
\hline Alicante & Murcia \\
\hline Autónoma de Barcelona & Navarra \\
\hline Autónoma de Madrid & Oviedo \\
\hline Barcelona & Politécnica de Catalunya \\
\hline Carlos III de Madrid & Pública de Navarra \\
\hline Castilla la Mancha & Sevilla \\
\hline Córdoba & U. a Distancia de Madrid \\
\hline Europea de Madrid Granada & Valladolid \\
\hline Granada & Zaragoza \\
\hline
\end{tabular}

En este caso, también han sido veinte universidades las que tienen a disposición de alumnos y profesores aplicaciones para los móviles de carácter educativo. La mayoría de las Universidades que se han lanzado a disponer de este tipo de servicios lo ha hecho para los dos principales sistemas operativos de móviles, algunas, de momento, sólo disponen de aplicaciones en uno de ellos.

Pero las aplicaciones institucionales que ponen a disposición de su equipo docente y alumnado las universidades españolas, no son las únicas que contribuyen a disponer de entornos educativos en los Smartphone. Las principales plataformas de educación virtual disponen de sus propias aplicaciones para estos dispositivos.

Moodle tiene en los mercados de IOS y de Android una aplicación estándar (My Moodle) desde las que los usuarios, cuyas universidades hayan creado su entorno virtual en Moodle, puedan acceder a su perfil, a sus cursos, etc. Por su parte, Blackboard cuenta con Blackboard Mobile Learn, que permite también el acceso a los usuarios de dicha plataforma a todo su contenido.

En ambos casos es necesario disponer de una versión actual del software instalada en el servidor de la aplicación. En el caso de Moodle se necesita una versión superior a 1.9, y en Blackboard a 8.0. Esto es un problema, ya que muchas de las Universidades aún disponen de versiones anteriores y están pendientes de actualización.

Pero las aplicaciones institucionales de las Universidades y las de las principales plataformas de entornos educativos virtuales no son las únicas que conviven y que tratan de facilitar la vida universitaria. Por ejemplo, bajo la categoría de educación en el App Store de Apple hay cerca de 92.000 aplicaciones para móviles. Este dato revela la magnitud de esta labor investigadora, lógicamente dentro de esta categoría no sólo se encuentran las aplicaciones para terminales móviles universitarias, también coexisten otro tipo de desarrollos sobre aprendizaje de idiomas, de educación primaria y secundaria, referenciadas a exámenes concretos, etc. 
Entre estos desarrollos destinados al ámbito universitario destaca iTunes U. Desde que en mayo de 2007 cinco universidades norteamericanas decidieran apostar por la plataforma de Apple y poner al alcance de todos los usuarios de iTunes material docente de forma gratuita, el crecimiento de esta herramienta ha sido importante. En 2010 más de 800 Universidades de todo el mundo utilizaban esta herramienta y compartían un total de 350.000 archivos, que supusieron más de 300 millones de descargas. En la actualidad estos datos han quedado obsoletos, ya que actualmente se ofrecen más de 500.000 cursos procedentes de instituciones educativas de 26 países diferentes.

El resto de aplicaciones de los principales mercados dedicadas al ámbito universitario se puede dividir en:

- Aplicaciones destinadas al docente. En este grupo destaca una aplicación llamada "Cuaderno del Profesor" desde la que el docente tiene toda la información de sus asignaturas, sus alumnos y sus clases, pudiendo ser actualizada en cualquier momento.

- Aplicaciones destinadas a los alumnos. Este es el grupo más numerosos y destacan las aplicaciones destinadas a la organización: creación de horarios, gestión de calendarios, agendas y tareas, etc.

- Otras aplicaciones. También hay una serie de aplicaciones que no pertenecen al ámbito docente pero que son muy útiles para esta labor. Por ejemplo: Dropbox (almacenamiento en la nube), Evernote (creación de notas) o DroidScan (escaneado de documentos mediante fotografías), entre otras.

Gracias a esta nueva forma de entender la labor docente dentro de la universidad, utilizando los nuevos recursos que nos brindan las nuevas tecnologías, las aplicaciones que aparecen y se desarrollan en el ámbito de la educación, se propagan por los Smartphone como lo podría hacer un virus por nuestro cuerpo, de forma casi incontrolada.

\section{Conclusiones}

Para esta labor investigadora se han analizado las principales herramientas de las que disponen las universidades españolas en tecnología móvil y se han alcanzado las siguientes conclusiones:

1. La labor educativa siempre implementa los nuevos avances tecnológicos para facilitar la transmisión de conocimientos. Aunque no siempre la implantación es descendente, en la actualidad, y gracias a que los alumnos, por su mejor adaptación a las nuevas tecnologías, ofrecen recursos novedosos a sus educadores utilizando los nuevos dispositivos móviles.

2. El desarrollo de Smartphone con la capacidad de conectarse a la red y de instalar aplicaciones está modificando la labor docente dentro de las universidades.

3. Las universidades españolas, conscientes de la importancia de adaptar las nuevas tecnologías a la educación, invierten parte de su capital en poner a disposición del personal docente y de los alumnos herramientas convergentes para alcanzar los objetivos educativos de cada materia. Este es el caso de los campus universitarios virtua- 
les, que según se ha analizado, utilizan todas y cada una de las universidades españolas. Actualmente dos plataformas son las que ofrecen mayoritariamente estas herramientas, el 77\%, y más concretamente el $58 \%$ de las universidades utilizan la plataforma de software libre Moodle para ofrecer unos servicios de aula virtual.

4. En este entorno analizado emergen con fuerza desarrollos lógicos adaptados a los nuevos dispositivos móviles de carácter educacional. En este sentido, se han desarrollado entornos universitarios mundiales conectados entre sí, es el caso de iTunes $\mathrm{U}$, una red de universidades en las que existe gran cantidad de cursos y recursos, cerca de 500.000, al alcance de cualquier persona con un Smartphone o una tableta de la compañía Apple conectada a internet.

5. En muchos casos la aplicación de este tipo de tecnologías se realiza de forma ascendente en lugar de ser descendente, es decir, los alumnos comienzan a utilizar las aplicaciones para facilitar su vida universitaria, y son los docentes los que tienen que formarse, bajo el criterio de sus alumnos, para adaptarlas a sus materias.

6. El número de aplicaciones para dispositivos móviles destinadas al ámbito educacional aumenta a diario y son utilizadas y creadas por las propias universidades. Actualmente 22 de las 77 universidades españolas disponen de una aplicación institucional en IOS o/y en Android que permite a los alumnos y profesores disponer de un espacio virtual en sus bolsillos en el que poder trabajar y comunicarse.

\section{Referencias bibliográficas}

APPLE (2012): “App Store”. En Apple: http://www.apple.com/es/iphone/from-theapp-store/. [Fecha de consulta: 15 de septiembre de 2012].

AREA MOREIRA, Manuel (2001): "Las redes de ordenadores en la enseñanza universitaria: Hacia los campus virtuales”, en GARCÍA-VALCÁRCEL, Ana: Didáctica universitaria. Madrid, La Muralla.

BUENGEEK (2011): "Cuantas aplicaciones existen en el mercado: Android vs Ios". En Buengeek: http://buengeek.com/cuantas-aplicaciones-existen-en-el-mercadoandroid-vs-iois. [Fecha de consulta: 14 de agosto de 2012].

DANIEL, John (1996): Megauniversities and Knowledge Media. London, Kogan Page.

EPSTEIN, Zach (2012): "Apple and Samsung to pull in 90\% of smartphone profits in 2012, UBS says". En BCR: http://www.bgr.com/2012/02/03/apple-and-samsung-to-pull-in-90-of-smartphone-profits-in-2012-ubs-says/. [Fecha de consulta: 15 de agosto de 2012].

FUNDACIÓN ORANGE (2011): "eEspaña. Informe anual sobre el desarrollo de la sociedad de la información en España 2011". http://www.informeeespana.es /docs/eE2011.pdf [Fecha de consulta: 10 de septiembre de 2012].

FUNDACIÓN TELEFÓNICA (2011): "La Información en España siE[11”. http://elibros.fundacion.telefonica.com/sie11/aplicacion_sie/ParteA/pdf/SiE_2011.pdf. [Fecha de consulta 10 de septiembre de 2012]. 
GARTNER (2012): "Worldwide Smartphone Sales Soared in Fourth Quarter of 2011 With 47 Percent Growth". http://www.gartner.com/it/page.jsp?id=1924314. [Fecha de consulta: 25 de agosto de 2012].

GOOGLE (2012): “Google Play”. En Google: https://play.google.com/store. [Fecha de consulta: 15 de septiembre de 2012].

INSTITUTO NACIONAL DE ESTADISTICA INE (2011): "Estadística de la enseñanza universitaria". En INE: http://www.ine.es/prensa/np655.pdf [Fecha de consulta: 23 de agosto de 2012].

MINISTERIO DE EDUCACIÓN CULTURA Y DEPORTE. (2012): "Universidades españolas". En Ministerio de Educación Cultura y Deporte: http://www.educacion.gob.es/educacion/universidades/educacion-superior-universitaria/que-estudiar-donde/universidades-espanolas.html. [Fecha de consulta: 12 de agosto de 2012].

\section{Dr. Héctor MOLINA GARCÍA}

Profesor responsable técnico del departamento de Tecnología de la Información de la Universidad Francisco de Vitoria.

Periodista

h.molina@ufv.es 